\section{P-124 A REVIEW ON HOW PATIENTS MANAGE WITH THEIR MEDICATION POST DISCHARGE FROM SAINT FRANCIS HOSPICE}

Jennifer Chan, Corinna Midgley. Saint Francis Hospice, Romford, UK

10.1136/bmispcare-2017-hospice.149

Background Research suggests that approximately 5\% of hospital admissions are medicines-related. There is an increased risk from errors or unintentional changes to a patient's medicines when their care is transferred. This includes when the patient is discharged from a hospice.

$\operatorname{Aim}(\mathbf{s})$ To identify whether patients are complying with their medicines and the types of medications related problems patients have post-discharge. To reconcile the medicines the patient is taking post-discharge against what had been prescribed on the inpatient unit.

Methods Patients deemed as medically fit by the medical team were approached by the hospice pharmacist and audit was explained to them. Those that wished to participate were contacted using their preferred method approximately two weeks post-discharge from the hospice. The data were collected using an adapted version of the medicines use review form used by community pharmacists.

Results

- A total of 20 patients were contacted

- $15 \%$ of patients had difficulties obtaining at least one of their prescribed medicines

- $5 \%$ of patients experienced at least one side effect from their medicines resulting in non-compliance

- $15 \%$ of patients chose to stop taking at least one of the prescribed medicine

- $10 \%$ of patients had medication changes made by the GP within two weeks post-discharge.

Conclusions This audit highlights some of the difficulties experienced by patients with their medication. Medicines prescribed by the team on the inpatient unit can often be quite specialised and not kept as stock in the community pharmacy causing patients to miss doses of critical medicines such as analgesics. Patients should receive counselling about their medicines before they are discharged to include information on who they should contact if they have problems with their medicines. A drug review post discharge from the hospice could also be beneficial,

\section{P-125 AUDITING FOR SUCCESS - DRIVING FORWARD QUALITY - ACHIEVING COMPLIANCE}

Juliana Luxton, Marissa Bravington-Turner. Thames Hospice, Windsor, UK

\subsection{6/bmjspcare-2017-hospice.150}

Using Hospice UK's audit tools Thames Hospice have been supporting our evidence gathering for CQC.

General Medicines Audit

Aim To use the template audit tool to assess the hospice's procedures, purchasing, storage and destruction, prescribing, administration of medicines. At Thames Hospice good clinical audit looks at an aspect of care from the patients' point of view, involves the patient wherever possible, and is multi-disciplinary, looking across all relevant professions and organisations. We asked:
- What should we be doing?

- Are we doing it?

- If not, how can we improve?

The Hospice UK audit tool helped us to think about our audit goals:

- Do we know and agree on what the best practice is?

- Will we be able to make any changes, if we find we need to?

- Will the changes make a difference to patients?

Methods Using the Hospice UK General Medicines Audit tool our small Audit team were easily able to review the hospice's procedures and practices regarding general medicines.

The audit:

- Looked at own practice

- Followed a systematic process

- Included standards to measure our practice against

- Involved everyone in the team and our patients

- Cross referenced standards against legislative and statutory requirements

Results The organisation completed a day-to-day general medicines audit on every patient across the medicines spectrum.

Conclusions Audit is an integral part of our hospice's ethos. Quality and assurance is intended, monitored and embraced throughout the organisation and supported us in improving training, practice and patient care.

\section{P-126 INTRODUCTION OF ELECTRONIC PRESCRIBING IN AN INDEPENDENT HOSPICE}

${ }^{1}$ Sarah Parnacott, ${ }^{2}$ Tracy Draper. ${ }^{1}$ Ashgate Hospicecare, Chesterfield, UK; ${ }^{2}$ Chesterfield Royal Hospital, Chesterfield, UK

\subsection{6/bmjspcare-2017-hospice. 151}

Aims To introduce successfully electronic prescribing in a hospice.

Background The hospice previously used paper drug cards to prescribe and record administration of medication. Our drugs for inpatient administration and for discharge prescriptions were supplied by a hospital four miles away. We had weekly inpatient support from a pharmacist one session a week and a pharmacy technician to reconcile medication against the patients' previous GP prescription and to check there were no prescribing errors. All requests for drugs had to be faxed along with the prescription card.

Method In February 2016 we introduced electronic prescribing to increase patient safety, reduce administration errors, increase efficiency in obtaining discharge prescriptions and reduce the time spent by nursing staff in ordering drugs. The nursing and medical staff received e-learning in order to become familiar with the prescribing and administration of medications. Four electronic carts were purchased at a cost of $£ 4000$ each. The pharmacist supported the implementation of the system undertaking drug rounds with nursing staff for two weeks. Nursing and medical staff who had previously worked in the hospital were already familiar with its use. The system is networked to the hospital so has the additional benefit of all prescriptions being visibly accessible by the hospital pharmacists.

Some challenges have been encountered particularly with regards to internet connectivity and the recent cyber attack but the system is backed up daily and we can resort to paper printouts of the electronic prescription. 
Results Results have shown a reduction in missed administration of drugs, a reduction in drug errors, both in prescribing and administration, increased speed of response for discharge prescriptions and a cost reduction in the pharmacy budget to the hospice.

Conclusion Electronic prescribing is a safe and efficient method for the prescribing and administration of drugs within an independent hospice.

\section{P-127 IMPROVING ADMINISTRATION TIME OF CONTROLLED DRUGS}

Jimmy Brash, Alison Bacon, George Coxon, Julie Cuckson, Andrew Fletcher, Tracy Parkinson, Debbie Seed, Barbara Sutton, Jayne Wilkinson. St Catherine's Hospice, Lancashire, UK

\subsection{6/bmispcare-2017-hospice.152}

Background In response to a perceived increase in patient needs and associated increase in workload, a simple analysis of nursing activity highlighted a need to revisit working practices and procedures. Of particular note for this poster was that each day 15 hours of registered nurse (RN) time was dedicated to the administration of 'as required'/PRN controlled drugs (CDs) along with additional (unmeasured) time required to administer those on a recurring prescription.

Aims

- Optimise symptom management

- Administer CDs alongside other medications

- Improve response time to requests for PRN CDs

- Create efficiencies in RN time management.

Methods

- Established a time limited working party to consider and modernise CD working practices. The group comprised:

- Two staff nurses

- Medical representation

- Ward manager and sisters

- CD Accountable Officer/registered manager

- Consider relevant aspects of The Misuse of Drugs Regulations 2001, Controlled drugs regulations and associated guidance

- Developed a robust and safe approach to CD management

- In conjunction with NHS England, a thorough risk assessment was completed.

Results A system has been devised which will make greater use of named patient CDs (oral/transdermal) rather than 'stock' CDs; this will enable:

- Storage of named patient CDs (regular prescriptions and PRN) in high quality medicines cupboards at each individual patient's bedside

- Secure and prompt access to the cupboards by registered nurses

- Single nurse administration of these CDs supported by a robust system of checks and balances

- Fully revised Standard Operating Procedures (CDs)

- Scope does not include parenteral medications.

Conclusion Benefits:

- Rapid access to PRN medications when needed

- Timely administration of regular prescription CDs and nonCDs; thus, a more responsive approach to much needed symptom management

- Reduced time locating stock cupboard keys

- Reduced risk of incorrect administration.

\section{P-128 A QUALITATIVE ANALYSIS OF THE EXPERIENCE OF EPISODIC BREATHLESSNESS IN ADVANCED LUNG CANCER}

${ }^{1}$ Emily Stowe, ${ }^{2}$ Richard Wagland. ${ }^{1}$ St Clare Hospice, Essex, UK; ${ }^{2}$ University of Southampton, Southampton, UK

\subsection{6/bmjspcare-2017-hospice.153}

Background Episodic breathlessness is a common and distressing symptom for people with advanced lung cancer, impacting carers as well as patients. Previous research has quantified the symptom occurrence of episodic breathlessness, identifying the average frequency, duration and severity of episodes, but rarely explored the lived experience of these different aspects. More understanding of the distress associated specifically with episodic breathlessness would inform more appropriate treatment strategies.

Aims To explore the nature of the distress associated with episodic breathlessness and the extent to which each aspect is bothersome for people with advanced lung cancer.

Methods Semi-structured interviews, analysed with an interpretative phenomenological approach, were conducted with four participants recruited from a hospice in Essex. All participants had advanced lung cancer. Ethical approval was secured from NRES Committee London - Hampstead (15/LO/1423) prior to the start of the study.

Results A multifaceted relationship existed between participants' perceptions of duration, frequency and severity in episodic breathlessness. The following key themes emerged: impact on self; searching for answers; impact of previous experience; emotional work of episodic breathlessness; difficulty in quantifying different aspects; identifying most bothersome aspects. Patients benchmarked severity against previous episodes. The unpredictability of severity and duration and constant assessment of potential triggers causing frequency engendered anxiety. Participants appeared to conflate severity and duration more than frequency, but one aspect often impacted a participant's perceptions of the other two.

Conclusions Findings suggest a complex, subjective relationship between different aspects of episodic breathlessness. The importance of considering the whole symptom experience, rather than just symptom occurrence is highlighted. The emotional work of episodic breathlessness should be considered in its management, as well as the interaction between different aspects. Clinicians should be aware of differences between individuals and seek to understand their lived experiences of episodic breathlessness to ensure appropriate treatment of this difficult symptom.

\section{P-129 THE USE OF RELAXATION, GUIDED IMAGERY AND VISUALISATION TECHNIQUES TO HELP RELIEVE ANXIETY AND PAIN IN PALLIATIVE CARE}

Jan Proud. St Margaret's Hospice, Somerset, UK

10.1136/bmjspcare-2017-hospice.154

Non-pharmacological interventions are not always considered as a primary or concurrent treatment for complex physical symptoms. Work carried out within the hospice setting has shown marked improvements in the physical and psychological condition of patients who have difficult to control symptoms, by using alternative patient-centred, holistic techniques. 